\title{
Systèmes de recommandation contextuels : Vers une typologie de contexte
}

\section{Context aware recommender systems - toward a context typology}

\author{
Elsa Negre \\ Paris-Dauphine University, PSL Research Universities, CNRS UMR 7243, LAMSADE, 75016 Paris, France \\ elsa.negre@dauphine.fr
}

\begin{abstract}
RÉSUMÉ. La montée en volume des données et informations de sources diverses exige un filtrage d'informations efficace afin d'être au plus près de l'utilisateur et répondre au mieux à ses besoins. Dans cet objectif, les systèmes de recommandation contextuels qui prennent en compte le contexte de l'utilisateur dans leur processus de recommandation, ont été proposés. Cependant il n'existe toujours pas de définition unique pour ce contexte. Dans cet article nous proposons une typologie du contexte utilisateur dans le cadre des systèmes de recommandation contextuels, afin de pallier les manques des précédentes propositions et répondre à un spectre assez large de cas d'application. En effet, cette typologie se veut générique avec une grande applicabilité. Nous montrons également comment l'utiliser, que ce soit dans la phase de recueil des informations contextuelles, celle de la modélisation ou celle de l'intégration au sein d'un système de recommandation contextuel.

ABSTRACT. The rise in volume of data and information from various sources requires effective information/data filtering to be closer to the user and best meet his/her needs. For this purpose, context-aware recommender systems that take into account the context of the user in their recommendation process, have been proposed. However, there is still no unique definition for context. In this article, we propose a typology of the user context for (context-aware) recommender systems, in order to overcome the shortcomings of the previous proposals and to answer a rather wide spectrum of application cases. Indeed, this typology is generic with great applicability. We also show how to use it, either in the phase of gathering contextual information, the modeling or the integration within a context-aware recommender system.

MOTS-CLÉS. Systèmes de recommandation, Contexte, Systèmes d'information, Aide à la décision

KEYWORDS. Recommender systems, Context, Information systems, Decision support systems
\end{abstract}

\section{Introduction}

La quantité d'information disponible sur le web est de plus en plus importante. Les utilisateurs peuvent facilement être envahis par ces données et informations. C'est alors que les techniques informatiques qui facilitent la recherche, l'extraction et le filtrage d'informations pertinentes paraissent nécessaires. L'une d'entre elles est la recommandation. Un système de recommandation propose à l'utilisateur des éléments qui sont susceptibles de l'intéresser. Beaucoup de systèmes de recommandation traditionnels, comme Amazon ou Netflix ont fait leurs preuves. Ils se basent essentiellement sur les notes attribuées par les utilisateurs aux éléments. Ces dernières années une nouvelle approche de recommandation a émergé, appelée recommandation contextuelle, qui a comme objectif d'être au plus près de l'utilisateur. En effet, on peut améliorer la pertinence des recommandations en prenant en compte des informations complémentaires, comme l'environnement et la situation actuelle dans laquelle l'utilisateur se trouve, ou plus précisément son contexte actuel. Des travaux comme ceux de (Riboni, Bettini, 2011) ont prouvé la corrélation entre le comportement d'un utilisateur et son contexte, d'où l'importance de l'intégration du contexte utilisateur dans le processus de recommandation.

Cependant la notion de contexte reste floue. En effet, à cause d'un manque de consensus, il n'existe toujours pas de définition unique pour le contexte.

L'intérêt du contexte dans les systèmes de recommandation n'a été constaté que très récemment (Baltrunas et al., 2012). En effet, les systèmes de recommandation traditionnels se basent seulement 
sur les utilisateurs et les éléments pour faire leurs recommandations, sans prendre en compte le contexte actuel de l'utilisateur. Cependant celui-ci peut influencer les intérêts de l'utilisateur, c'est pourquoi il est important de prendre en compte ce type d'informations complémentaires afin d'obtenir des recommandations plus appropriées (Baltrunas et al., 2012).

L'objectif de cet article, qui étend (Negre, 2018), est d'améliorer la représentation du contexte de l'utilisateur dans le cadre des systèmes de recommandation (contextuels), en proposant une typologie de contexte sous la forme d'une catégorisation hiérarchique des facteurs de contexte. Cette typologie se veut générique et applicable dans de nombreux domaines. Cela est un pas vers l'amélioration des systèmes de recommandation.

Cet article est organisé ainsi : nous présentons dans la section 2, la notion de contexte, puis dans la section suivante, notre typologie de contexte. La section 4 illustre l'applicabilité et la généricité de notre typologie de contexte. Enfin, nous concluons et énonçons quelques perspectives de recherche en section 5.

\section{Contexte}

Malgré de bonnes performances des systèmes de recommandation, les recommandations ne sont parfois « pas assez pertinentes ». Par exemple, supposons que recommander un film à un jeune homme de 20 ans consiste à lui proposer des films de guerre ou d'action et supposons que lorsqu'il est avec une amie, il préfère qu'on lui recommande des films romantiques. Ici le contexte/la situation ${ }^{1}$ influence les préférences, les envies et les intérêts des utilisateurs et de ce fait, leurs décisions. Nous souhaitons donc intégrer les données/informations (qu'elles soient qualitatives et/ou quantitatives) contextuelles au système de recommandation qui devient un système de recommandation contextuel.

La notion de «contexte » a été étudiée dans divers domaines, notamment en informatique ubiquitaire (pervasive and ubiquitous computing) (Riboni, Bettini, 2011; Henricksen, Indulska, 2006; Schilit, Theimer, 1994) mais il est difficile d'établir une définition standard (unique) en raison de la nature multiforme du contexte (Bazire, Brézillon, 2005). La définition la plus acceptée est celle de (Dey et al., 2001) qui définit le contexte comme toute information qui peut être utilisée pour caractériser la situation d'une entité. Une entité peut être une personne, un lieu ou un objet qui est considéré pertinent dans l'interaction entre un utilisateur et une application, tout en incluant ces deux derniers. Afin de connaître le contexte, il faut collecter les informations contextuelles. Le contexte peut être capturé, collecté explicitement ou implicitement (Mostefaoui et al., 2004).

Les systèmes de recommandation traditionnels se basent seulement sur les utilisateurs et les éléments pour faire leurs recommandations, alors que les systèmes de recommandation contextuels, quant à eux, prennent en compte le contexte de l'utilisateur qui peut influencer les intérêts/besoins de l'utilisateur. Ainsi, les systèmes de recommandation contextuels existants sont plus efficaces/pertinents (c'est-à-dire plus en adéquation avec les besoins de l'utilisateur) que les systèmes de recommandation traditionnels (Adomavicius, Tuzhilin, 2011). Cependant, leur performance est impactée par le contexte, qui est flou et omniprésent (Bazire, Brézillon, 2005).

1. En science de l'information, les notions de contexte et situation sont utilisées de manière interchangeable. (Cool, 2001) suggère que les contextes sont les grandes lignes, c'est-à-dire l'environnement statique, et les situations sont les environnements dynamiques dans lesquels les processus d'interprétation se déroulent. 
Notre objectif est d'aller vers une modélisation du contexte pour une meilleure prise en compte de celui-ci. Pour atteindre cette modélisation, dans (Ferdousi et al., 2017), à partir d'une étude bibliographique, nous avons identifié dans un premier temps tous les facteurs de contexte qui ont été étudiés.

Par ailleurs, la modélisation du contexte reste encore compliquée compte tenu de la nature des données et/ou informations contextuelles : en effet le modèle doit pouvoir gérer les sources de données variées, l'hétérogénéité au niveau de leur qualité et de leur durée de vie et la nature imparfaite de cellesci (Henricksen, Indulska, 2006). Il existe différents modèles de contexte (Bettini et al., 2010) : attribut/valeur, mots clés, graphes, logique, ...

Il est à noter que dans cet article, nous abordons la représentation, la modélisation du contexte ainsi que son intégration au sein du système de recommandation.

Traditionnellement, le problème de la recommandation peut être résumé comme le problème d'estimation des scores pour les éléments qui n'ont pas encore été vus par un utilisateur, c'est-à-dire un problème de prédiction où le système de recommandation prédit les scores/évaluations de l'utilisateur en fonction du profil de l'utilisateur, il s'agit d'une fonction telle que $: r_{R S}:$ Utilisateurs $\times$ Elements $\rightarrow$ Scores (Adomavicius, Tuzhilin, 2011). Avec le contexte, cette fonction d'évaluation des scores pour les systèmes de recommandation contextuel devient $: r_{C A R S}:$ Utilisateurs $\times$ Elements $\times$ Contextes $\rightarrow$ Scores (Adomavicius, Tuzhilin, 2011). En outre, plusieurs techniques existent pour l'intégration du contexte dans le processus de recommandation : (i) le pré-filtrage, où seules les données qui correspondent au contexte sont sélectionnées (comme entrées), puis une méthode de recommandation traditionnelle est appliquée sur celles-ci, (ii) le post-filtrage, où une méthode de recommandation traditionnelle est appliquée sur la totalité des données, puis les résultats de la recommandation (sorties) sont ajustés en fonction du contexte, et (iii) le context modeling dans lequel les données et informations contextuelles sont directement prises en compte dans le processus de recommandation (les étapes) comme une dimension supplémentaire (Adomavicius, Tuzhilin, 2011).

\section{Les facteurs de contexte de l'utilisateur}

Afin de décrire plus concrètement en quoi consiste le contexte d'un utilisateur, de multiples catégorisations de facteurs de contexte ont été proposées (Ingwersen, Järvelin, 2005). A partir d'une étude bibliographique $^{2}$ (dont une partie est synthétisée dans le tableau 1) et des facteurs de contexte présentés par (Adomavicius, Tuzhilin, 2011), nous avons regroupé/recoupé et structuré les travaux existants. Puis nous avons proposé une catégorisation hiérarchique, comme illustrée sur la figure 1 (Ferdousi et al., 2017). Cette catégorisation hiérarchique est un arbre, c'est-à-dire un graphe non orienté, acyclique et connexe, vu comme la généralisation de listes (puisque les listes peuvent être représentées par des arbres) favorisant ainsi son utilisation. Nos objectifs pour cette nouvelle proposition de catégorisation des facteurs de contexte sont de répondre aux besoins des systèmes de recommandation contextuels tout en (i) satisfaisant ${ }^{3}$ la définition de (Dey et al., 2001), (ii) compensant les lacunes (non-satisfaction de la définition

2. Il est à noter que les facteurs "Individualité / Profil utilisateur" et "Localisation" sont les deux seuls facteurs de contexte utilisés par tous. Il est également important de remarquer qu' aucun des travaux ne prend en compte tous les facteurs. Cela vient du fait que selon les domaines d'application, certains facteurs de contexte sont considérés plus utiles que d'autres.

3. Les caractéristiques des lieux, des personnes et des objets qui jouent un rôle dans l'interaction entre l'utilisateur et l'application sont respectivement représentés par les unités du contexte physique, par l'unité sociale du contexte utilisateur et par l'unité équipement du contexte physique. Les caractéristiques de l'utilisateur et de l'application apparaissent dans les contextes personnel et technique. 
de (Dey et al., 2001) ou caractérisation non optimale) des précédentes propositions, (iii) permettant de travailler le contexte sur plusieurs niveaux ${ }^{4}$ et (iv) permettant de l'appliquer sur un spectre assez large de cas d'applications ${ }^{5}$.

\begin{tabular}{|c|c|c|c|c|c|c|c|}
\hline & 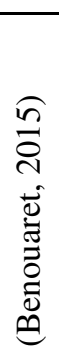 & 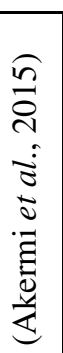 & 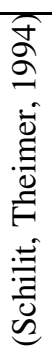 & 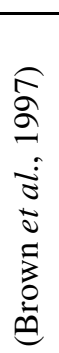 & 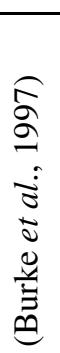 & 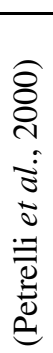 & 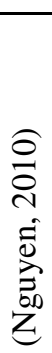 \\
\hline Temps & $\mathrm{X}$ & $\mathrm{X}$ & & $\mathrm{X}$ & & & $\mathrm{X}$ \\
\hline Individualité / Profil d'utilisateur & $\mathrm{X}$ & $\mathrm{X}$ & $\mathrm{X}$ & $\mathrm{X}$ & $\mathrm{X}$ & $\mathrm{X}$ & $\mathrm{X}$ \\
\hline Activité & $\mathrm{X}$ & $\mathrm{X}$ & & & & & $\mathrm{X}$ \\
\hline Relations & $\mathrm{X}$ & & & & & & $\bar{X}$ \\
\hline Localisation & $\mathrm{X}$ & $\mathrm{X}$ & $\mathrm{X}$ & $\mathrm{X}$ & $\mathrm{X}$ & $\mathrm{X}$ & $\mathrm{X}$ \\
\hline Objet & & & $\mathrm{X}$ & & $\mathrm{X}$ & $\mathrm{X}$ & $\mathrm{X}$ \\
\hline Saison & & & & $\mathrm{X}$ & & & \\
\hline Température & & & & $\mathrm{X}$ & & & \\
\hline Contexte social & & & & & & $\mathrm{X}$ & $\bar{X}$ \\
\hline Contexte matériel & & & & & & $\mathrm{X}$ & $\mathrm{X}$ \\
\hline
\end{tabular}

Tableau 1. Extrait de facteurs de contexte présents dans la littérature

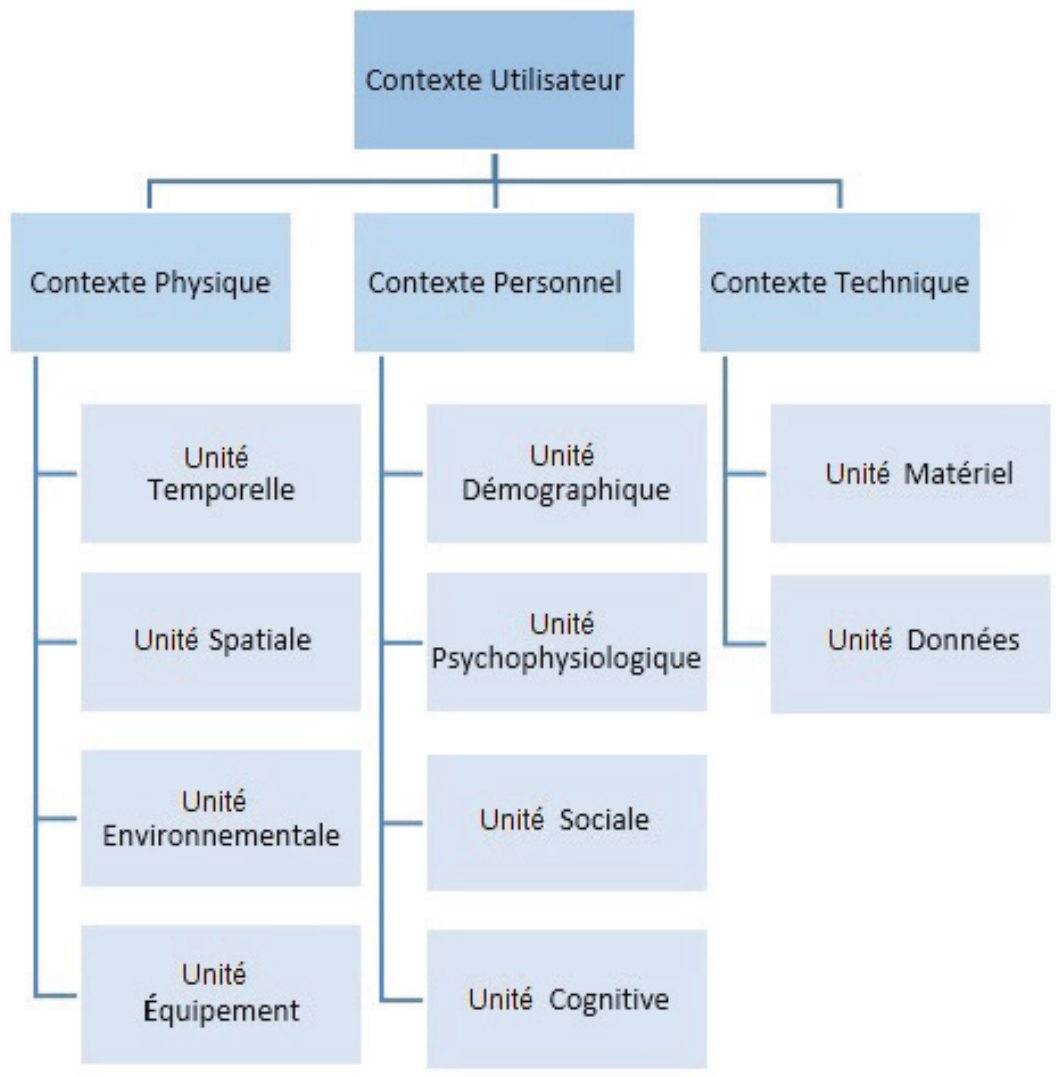

Figure 1. Catégorisation des facteurs de contexte

\footnotetext{
4. Notre catégorisation à plusieurs niveaux peut s'adapter à différents cas d'applications et à leurs besoins, et permet de travailler le contexte de façon plus générale ou plus fine.

5. Nous espérons tendre vers une représentation, rassemblant tous les facteurs de contexte possible, pour une application au plus grand nombre de domaines.
} 
Notre catégorisation a comme principales familles de contexte : le contexte physique, le contexte personnel et le contexte technique (Ferdousi et al., 2017). Le contexte utilisateur est alors l'union de toutes ces familles de contexte, chacune composée de plusieurs unités ${ }^{6}$ :

1. Le contexte physique regroupe tous les aspects sur lesquels la position géographique de l'utilisateur va avoir une forte influence. Nous avons regroupé plusieurs unités dans cette catégorie :

(a) L'unité temporelle : comme le moment de la journée, semaine/weekend, la saison, les évènements (anniversaire, nouvel an, ...),

(b) L'unité spatiale : qui selon le cas d'application peut être représentée par la position géographique exacte (coordonnées GPS, longitude/latitude) ou par des classes nominales (pour dire si l'utilisateur est chez lui, au travail, en voyage, ...).

(c) L'unité environnementale : en fonction du cas d'application, cette unité peut représenter des caractéristiques environnementales comme la température, la météo, le niveau de luminosité ou le niveau sonore du lieu où l'utilisateur se trouve, et/ou la situation régionale de ce lieu, comme une guerre, une catastrophe naturelle, une crise économique, ...

(d) L'unité équipement : tout ce (non humain : objet ou espace) qui entoure l'utilisateur comme : barbecue, ustensiles, électroménager (friteuse, four, ...), imprimante, jardin/terrasse, ...

2. Le contexte personnel représente les informations plus spécifiques de l'utilisateur qui sont regroupées en quatre unités :

(a) L'unité démographique regroupe notamment les informations sur l'identité (le nom, l'âge, le sexe, la nationalité, ...) de l'utilisateur.

(b) L'unité sociale fait référence à la présence et au rôle des autres personnes autour de l'utilisateur. Selon le cas d'application, on peut prendre en compte seulement les personnes présentes pendant l'utilisation de l'application par l'utilisateur, les personnes avec lesquelles on veut partager l'activité en question, ou bien aller plus loin en prenant en compte les relations plus fines, comme les amis, la famille, les collègues, les voisins, ...

(c) L'unité psychophysiologique représente l'aspect psychophysiologique de l'utilisateur, comme son état d'esprit, son humeur, son degré de fatigue, ...

(d) L'unité cognitive fait référence aux expériences de l'utilisateur, ses objectifs, ses contraintes, son activité, ...

3. Le contexte technique représente les caractéristiques des dispositifs utilisés par l'utilisateur. Cela peut être :

(a) Le matériel utilisé par l'utilisateur pour accéder au système de recommandation contextuel, comme le dispositif utilisé, les processeurs, la capacité du réseau, ...

(b) Les données qui sont manipulées par l'application, leur format (texte, film, audio, image, ...), leur sources de provenance, la qualité de ces données, leur période de validité, leur exactitude,

Il est à noter que chaque unité sera instanciée en fonction du cas d'application, de la pertinence et de la disponibilité des indicateurs associés.

6. Nous sommes conscients que notre catégorisation en trois grandes familles est discutable, mais comme toute catégorisation hiérarchique, il est possible de grouper/dissocier certaines familles/unités afin d'avoir un plus grand/petit nombre de familles/unités. 
Finalement, nous souhaitons aller plus loin qu'une nouvelle catégorisation en proposant notre catégorisation (Ferdousi et al., 2017) comme une typologie, i.e. un système de classification générique et standard pour les données/informations contextuelles dans les systèmes de recommandation. A cet effet, dans la section suivante, nous montrons l'applicabilité de notre typologie sur trois domaines d'application avec des enjeux et des données différents.

\section{Applications}

Ainsi, nous précisons dans cette section comment instancier notre catégorisation du contexte dans trois domaines d'application : OLAP (OnLine Analytical Process), un environnement commercial, et la gestion de crise. Nous les présentons en fonction de la densité des informations contextuelles, c'est-à-dire de la quantité de familles/unités de contexte prises en compte.

\subsection{En OLAP}

Recommander une requête OLAP à un décideur d'une grande entreprise française consistera à retourner des requêtes relatives au chiffre d'affaire annuel de la société. Mais si la société s'ouvre à l'international (États-Unis par exemple) ou si certaines données (anciennes) ont été archivées alors le décideur préfèrerait que le système lui recommande des requêtes « récentes » ou en rapport avec les États-Unis. En OLAP, les systèmes de recommandation prennent souvent un log parmi leurs données d'entrée. Or, les analyses OLAP (stockées dans le log) sont lancées régulièrement mais cette régularité peut être annuelle. Donc, le log peut contenir des données très anciennes sans rapport avec les analyses réalisées au moment de l'utilisation par le système de recommandation. De sorte que le système de recommandation retourne des recommandations non pertinentes.

Prendre en compte des informations contextuelles permettrait de pallier ce manque de pertinence.

\subsubsection{Familles et unités de contexte}

Nous avons commencé par déterminer quelles informations contextuelles sont utiles pour les systèmes de recommandation en OLAP (Negre, 2017). En effet, dans (Negre, 2017), cinq critères pertinents de contexte ont été détectés : Temps, Individualité/Profil Utilisateur, Activité, Relations et Contexte matériel. Nous les présentons ici selon la catégorisation des facteurs de contexte présentée précédemment. Il est à noter que les familles/unités de contexte, qui n'apparaissent pas, ont été considérées comme inutiles en fonction des spécificités d'OLAP.

\subsubsection{Contexte Physique}

- Unité temporelle : relative au critère Temps de (Negre, 2017).

\subsubsection{Contexte Personnel}

- Unité démographique : relative au critère Individualité/Profil Utilisateur.

- Unité sociale : relative au critère Relations.

- Unité cognitive : relative au critère Activité. 


\subsubsection{Contexte Technique}

- Unité Matériel : relative au critère Contexte matériel.

Finalement, ici, chaque critère de (Negre, 2017) est associé à une unité de contexte différente.

\subsubsection{Modélisation}

Une modélisation des cinq critères pertinents a été proposée dans (Negre, 2017) sous la forme d'une ontologie générale (ontologie de domaine) comme illustré par la figure 2a (par souci de lisibilité, nous nous limitons à afficher le premier niveau). D'après (Soualah Alila, 2015), qui a fait une comparaison entre les différents modèles de contexte, le modèle ontologique permet une bonne validation partielle des données et une bonne formalisation du modèle.

Passer de la catégorisation en cinq critères de (Negre, 2017) à notre proposition n'a pas d'impact sur la modélisation puisque chaque critère de (Negre, 2017) a été associé à une seule unité de contexte (seuls les intitulés changent comme illustré par la figure $2 b$ ).

\subsubsection{Intégration}

Comme évoqué en introduction, le contexte de l'utilisateur peut être incorporé à différents niveaux du processus de recommandation : (i) en pré-filtrage, (ii) en post-filtrage, et (iii) dans la phase de génération/calcul des recommandations (contextual modeling) (Adomavicius, Tuzhilin, 2011). De plus, (Panniello, Gorgoglione, 2011) conclut que le post-filtrage semble être le plus efficace. Or, à notre connaissance, il n'existait pas de système de recommandation contextuel dans le domaine des entrepôts de données, et en particulier de l'analyse en ligne (OLAP). Aussi, dans (Negre et al., 2018), nous avons proposé un tel système à base de post-filtrage pour recommander des requêtes OLAP.

En effet, afin de faciliter l'exploration de l'utilisateur lors de l'analyse en ligne, des systèmes de recommandation sont développés. Cependant, certaines recommandations peuvent être inappropriées (requêtes non pertinentes ou non calculables). Pour remédier à ces disparités, nous avons proposé d'intégrer les données contextuelles dans le système de recommandation. Comme indiqué précédemment, en OLAP, le contexte de l'utilisateur est modélisé via une ontologie contenant cinq catégories : Temps, Individualité, Activité, Relations et Contexte Matériel. Notre approche de post-filtrage associe un processus de recommandation traditionnel à un processus de contextualisation dans lequel les requêtes recommandées retournées par le système de recommandation traditionnel sont contextualisées afin d'obtenir des requêtes recommandées tenant compte du contexte. La figure 3 illustre notre approche où, au cours du processus de recommandation, la requête OLAP, $q$, lancée par le décideur (i) est enregistrée, (ii) permet de mettre à jour le contexte du décideur et (iii) correspond à l'entrée du système de recommandation (traditionnel). Ensuite, le système de recommandation basé sur un journal/log renvoie un ensemble de requêtes recommandées $Q_{R}=\left\{q_{i}\right\}$. Puis commence le processus de contextualisation où chaque requête $q_{i}$ de $Q_{R}$ est traitée :

- la requête $q_{i}$ peut être traitée et n'est pas obsolète; dans ce cas, cette requête est ajoutée directement à l'ensemble résultat, $Q_{R_{c x t}}$,

- la requête $q_{i}$ est obsolète, auquel cas cette requête nécessite certaines mises à jour en fonction d'un contexte donné et la nouvelle requête sensible au contexte $q_{i_{c x t}}$ est ajoutée à $Q_{R_{c x} t}$. 


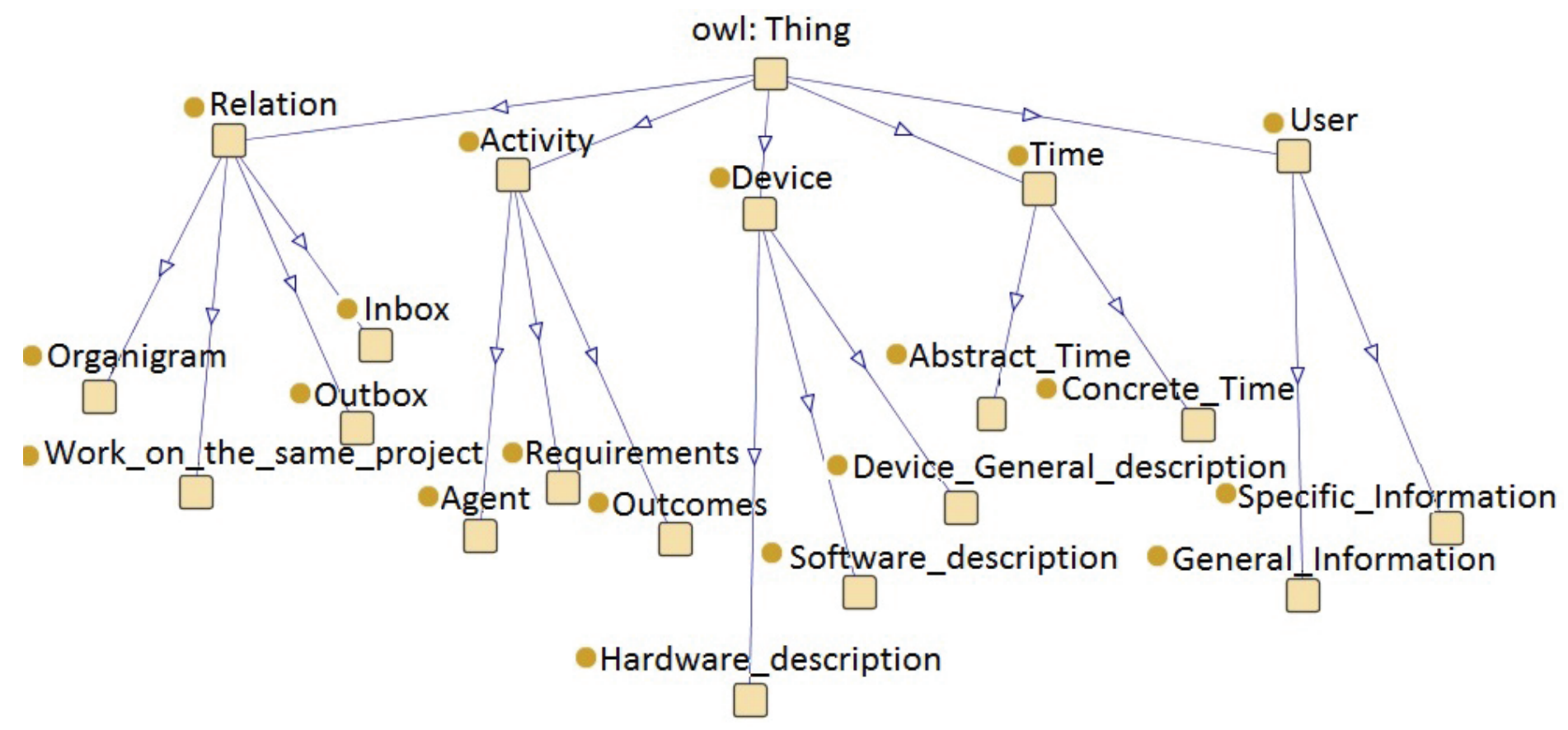

(a) Ontologie (cinq critères) de (Negre, 2017)

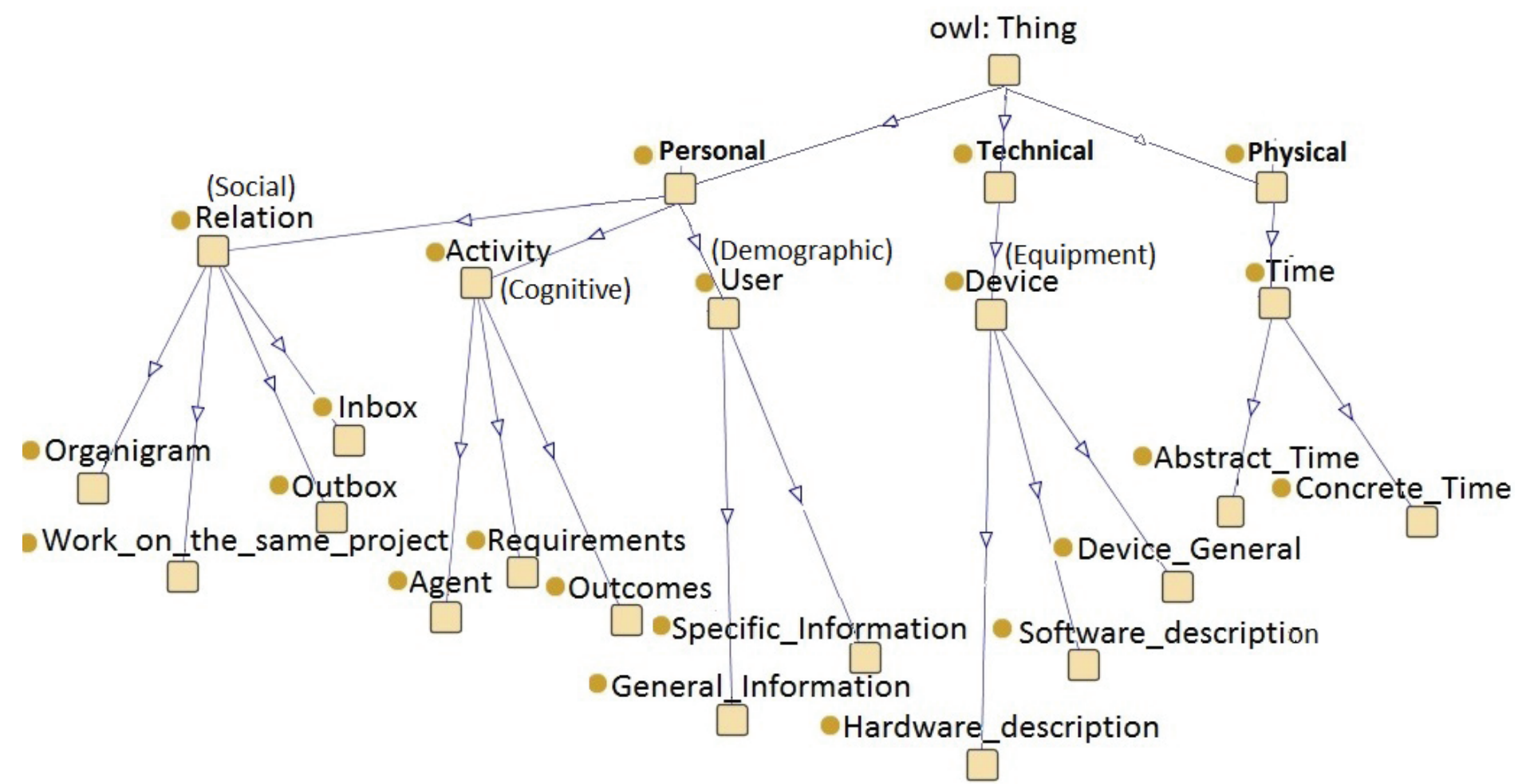

(b) Ontologie avec notre typologie

Figure 2. Ontologie générale de contexte dans le cadre d'un système de recommandation de requêtes OLAP.

Enfin, cet ensemble $Q_{R_{c x t}}=\left\{q_{i}\right\} \cup\left\{q_{i_{c x t}}\right\}$ de recommandations tenant compte du contexte est retourné au décideur.

Plus précisément, lorsque une requête $q$ recommandée par le système de recommandation traditionnel est obsolète (trop ancienne pour être pertinente par exemple), notre approche modifie la requête $q$ grâce aux données contextuelles. Si la requête $q$ contient une sélection à des niveaux en rapport avec le temps, les valeurs correspondantes sont remplacées/mises à jour en fonction des informations contextuelles de 


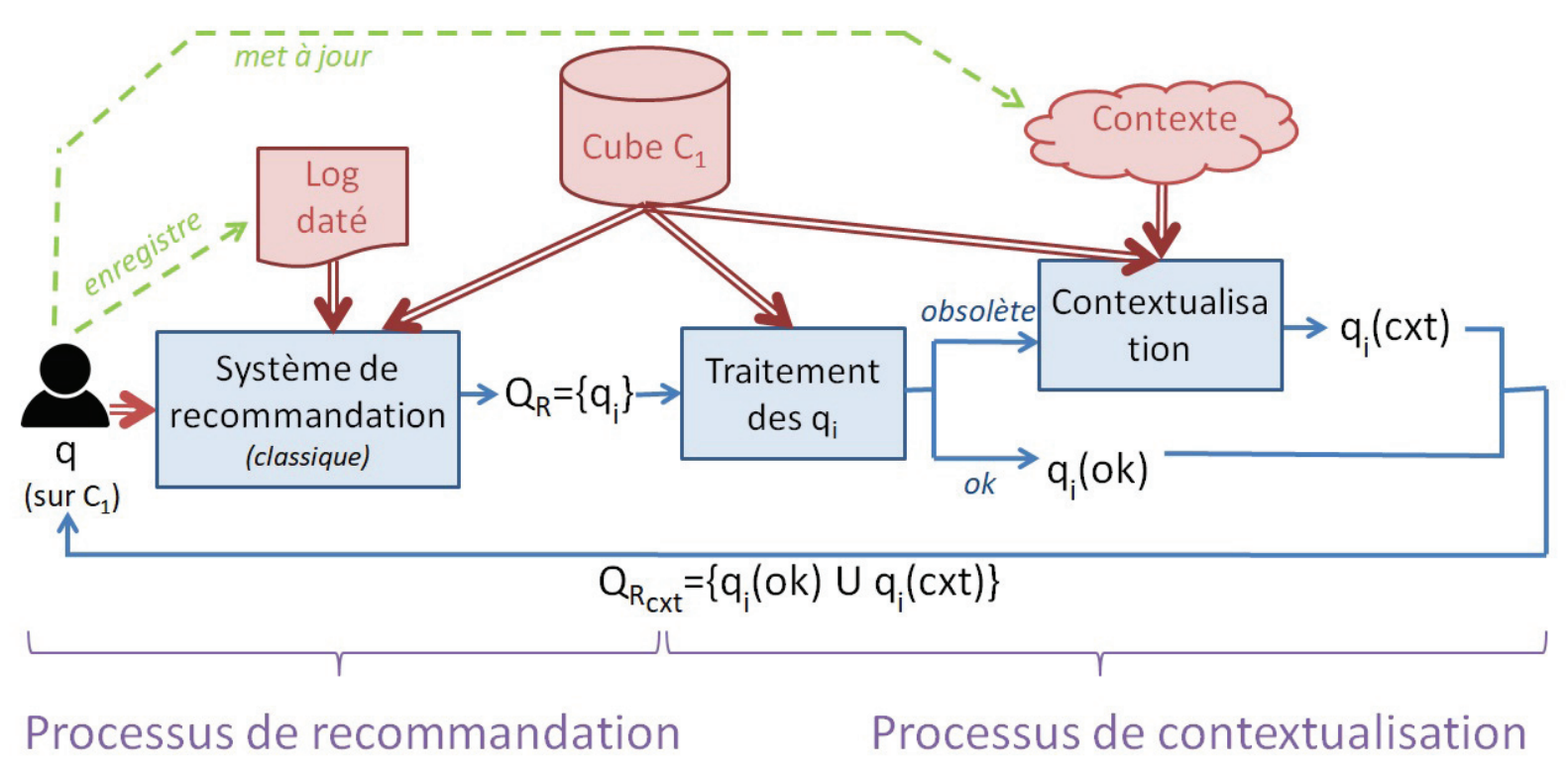

Figure 3. Un système de recommandation contextuel pour OLAP. Les flèches doubles sont les entrées et les flèches pleines sont des exécutions

l'unité de contexte Temps, en gardant le laps de temps écoulé entre le moment où la requête $q$ a été lancée et les valeurs temporelles des sélections. Si la requête $q$ ne contient pas de sélection temporelle, l'algorithme de clustering, k-medoid, est utilisé pour partitionner les requêtes les plus récentes du log (en fonction d'un certain seuil). Chaque cluster $c l_{j}$ est représenté par une requête spécifique $: q_{j}^{\text {med }}$. Puis la requête $q$ est remplacée par la requête $q_{j}^{\text {med }}$ qui lui est la plus similaire. Si (et seulement si) la requête contextuelle obtenue est calculable et peut être affichée (en fonction de l'unité de contexte, Contexte Matériel), elle est retournée par le système comme recommandation contextualisée.

Par ailleurs, si une requête $q$ n'est pas calculable, et qu'il s'agit d'un problème de schéma, c'est-à-dire que certains niveaux hiérarchiques n'existent pas ou ne sont pas accessibles mais que la dimension correspondante existe, alors, la requête $q$ remonte (roll-up) jusqu'au niveau 'ALL' de cette dimension. Si le problème de schéma est plus conséquent, c'est-à-dire, par exemple, que certaines dimensions n'existent pas ou ne sont pas accessibles, alors toutes les données relatives à cette dimension sont supprimées de la requête $q$ et des contraintes sont ajoutées grâce notamment à l'unité de contexte Individualité. S'il s'agit d'un problème de données, c'est-à-dire que des valeurs de niveaux n'existent pas mais que le niveau existe, alors, toutes les valeurs existantes de ce niveau sont affichées et des contraintes sont ajoutées grâce aux unités de contexte Individualité et/ou Temps. Si (et seulement si) la requête contextuelle obtenue est calculable et peut être affichée (en fonction de l'unité de contexte, Contexte Matériel), elle est retournée par le système comme recommandation contextualisée.

A partir des tests réalisés, il apparaît que notre approche permet au système de proposer des recommandations plus pertinentes et qui tiennent compte du contexte.

Nos perspectives de recherche incluent la réalisation d'expériences sur des données réelles. De plus, notre système de recommandation contextuel devra être applicable à la fois sur des données détaillées et sur des données agrégées. Enfin, d'autres approches peuvent être proposées pour modéliser et intégrer le contexte dans les systèmes de recommandation contextuels pour OLAP, en tenant en compte, par exemple, de la nature multidimensionnelle du contexte. 


\subsection{Dans un environnement commercial}

Nous nous sommes intéressés aux données/informations contextuelles dans un environnement commercial particulier : celui de la vente de véhicules d'occasion (Arduin et al., 2014). Il est à noter que nous avons commencé à partir d'observations « terrain » et que l'approche proposée est spécifique au cas rencontré.

\section{Acheter un véhicule d'occasion}

En France, en général, pour vendre un véhicule à un distributeur, ce véhicule doit être en bon état et pas trop vieux. Le distributeur achète votre véhicule uniquement si vous achetez un nouveau dans son entreprise. Il peut se permettre d'offrir un prix d'achat plus élevé car il fera une marge bénéficiaire significative avec le véhicule que vous achèterez. La promesse de la société sur laquelle porte notre étude, est d'acheter tous les véhicules sans condition, quel que soit leur état et sans obligation d'acheter un autre véhicule.

\section{Vendre un véhicule d'occasion}

Lorsqu'une personne (privée), Elsa, veut vendre son véhicule, elle demande une évaluation préalable du véhicule d'occasion sur le site de la société. Elle donne des informations sur les principales caractéristiques du véhicule telles que la marque, le modèle et l'âge. À la suite de cette requête, elle obtient une évaluation du prix d'achat du véhicule. Le système ne prend pas en compte l'usure du véhicule et il estime que le véhicule est en bon état. Lors de l'interrogation pour obtenir une première évaluation sur le site web, Elsa renseigne son adresse et son numéro de téléphone, elle sera contactée par la société pour prendre rendez-vous dans une agence. Quand Elsa va à l'agence, elle est reçue par un expert automobile, John. Il examine le véhicule et évalue les coûts pour reconditionner le véhicule. Cette étape est très importante car elle contrôle l'état de fonctionnement du véhicule, John doit faire un essai routier et identifier d'éventuelles anomalies. Les données sont intégrées et stockées via une plateforme informatique afin de demander aux experts en cotation une cotation du véhicule. La valeur que John obtiendra du service des achats à travers cette plateforme sera la meilleure proposition et John n'aura aucune marge de manœuvre sur cette proposition ${ }^{7}$.

\subsubsection{Familles et unités de contexte}

Grâce à des entretiens et des échanges avec des experts et des vendeurs, il apparait que la prise en compte du contexte améliorerait l'évaluation du prix du véhicule ainsi que le succès de la transaction. Ainsi, quatre critères ont été détectés comme utiles : la localisation du véhicule, le marché, les coûts de réparation et le contexte personnel d'une vente particulière. Nous les présentons ici selon notre typologie :

7. La façon dont les véhicules sont évalués n'est pas discutée ici. Cependant, cette évaluation est imprévisible parce qu'elle dépend de chaque expert en cotation. Pour mieux estimer le marché, ces experts utilisent différents sites publicitaires vendant des véhicules sur le web et examinent les prix. Ils évaluent le véhicule de sorte qu'il soit bien placé parmi les annonces similaires. Cette approche conduit parfois à ce que le prix d'achat offert au particulier ne soit pas conforme au prix réel de revente. 


\subsubsection{Contexte Physique}

- Unité spatiale : La localisation du véhicule. Par exemple, les experts savent que certains véhicules seront vendus différemment en fonction de leur situation géographique.

- Unité environnementale : Le marché. Par exemple, si un véhicule est très populaire, la société le gardera en stock moins de temps, donc ce sera moins cher. Cependant, si un véhicule n'est pas populaire, il se passera plus de temps entre l'achat du véhicule et sa revente, il y a donc un coût de stockage supplémentaire pour la société.

- Unité Équipement : Les coûts de réparation. Par exemple, une simple égratignure peut provoquer le remplacement d'une partie importante du véhicule et seul un expert peut le savoir.

\subsubsection{Contexte Personnel}

- Unités démographique, psychophysiologique, sociale et cognitive : Le contexte personnel d'une vente particulière. Par exemple, les problèmes financiers ou familiaux peuvent engendrer un besoin urgent de vendre le véhicule quel que soit le prix ou bien, cela peut entraîner une tentative de négociation du prix aussi élevé que possible.

Finalement, ici, trois critères de (Arduin et al., 2014) sont associés, chacun, à une unité de contexte et un critère est associé à une famille de contexte.

\subsubsection{Modélisation}

Nous avons proposé de modéliser ce contexte en combinant une approche d'aide à la décision multicritère et une approche coût-bénéfice dans (Arduin et al., 2014). Les « meilleures » décisions possibles obtenues avec notre modèle pourront ensuite être utilisées par le système de recommandation comme des données/sources externes complémentaires.

Cette approche combinée est illustrée sur la figure 4a selon une hiérarchie de critères. Étant donné que les données relatives au coût-bénéfice n'étaient pas fournies par la société, nous avons modélisé uniquement, dans cette hiérarchie, le nœud associé aux facteurs de contexte.

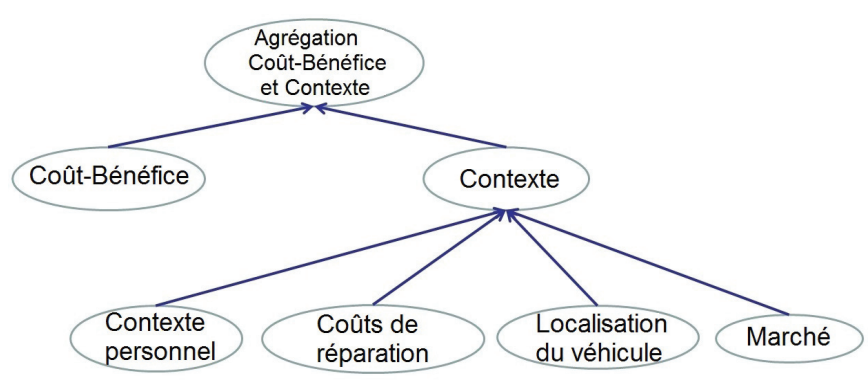

(a) SANS la typologie de contexte

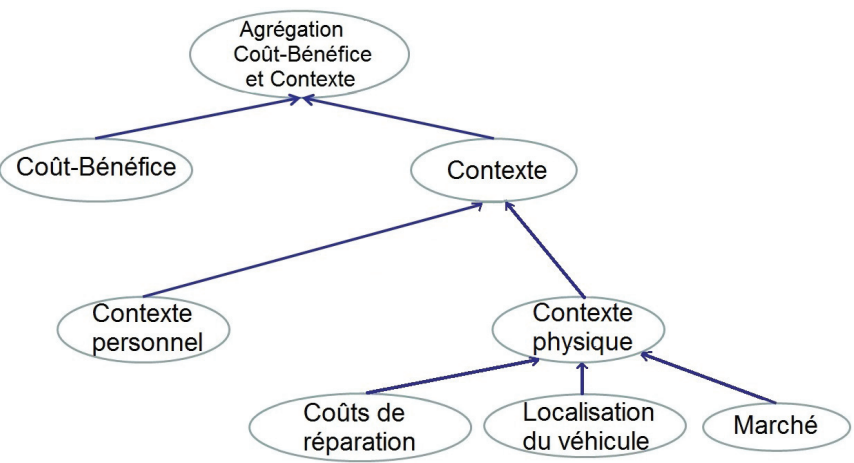

(b) AVEC la typologie de contexte

Figure 4. Un modèle hiérarchique d'aide à la décision multicritère vu comme une agrégation des coûts-bénéfices et du contexte.

Passer de la catégorisation en quatre critères de (Arduin et al., 2014) à notre proposition n'a pas d'impact important sur la modélisation. En effet, le critère Contexte personnel est associé à une seule famille de contexte et les trois autres critères sont associés à trois unités de contexte différentes d'une même 
famille. Le modèle hiérarchique de la figure $4 \mathrm{a}$ deviendrait celui de la figure $4 \mathrm{~b}$ permettant de faire apparaitre une niveau intermédiaire dans la hiérarchie mais qui n'aurait pas d'impact sur l'analyse multicritère car celle-ci utilise des critères détaillés.

\subsubsection{Intégration}

Parmi les méthodes permettant d'intégrer les informations contextuelles dans le processus de recommandation (pré-filtrage, post-filtrage ou modélisation contextuelle (contextual modeling)), il n'y a pas de grand gagnant (Panniello et al., 2009), et les expériences conduites ont montré que l'efficacité de la méthode choisie dépend du jeu de données et du domaine d'application. À l'instar des approches de post-filtrage, l'un des avantages des approches de pré-filtrage par rapport aux approches de modélisation contextuelle est la possibilité de réutilisation des techniques de recommandation traditionnelles. Les techniques de post-filtrage se caractérisent par le fait qu'une recommandation sans contexte est d'abord calculée puis, une réorganisation ou un filtrage est effectué sur la liste de recommandations en tenant compte des informations contextuelles. Donc, une grande partie de la recommandation a été faite sans prendre en compte les informations contextuelles. Au lieu de cela, dans les approches de pré-filtrage, les informations contextuelles de l'utilisateur sont placées au premier plan et sont utilisées lors de la première étape du processus pour guider la recommandation. C'est la raison pour laquelle les approches de pré-filtrage ont attiré de nombreux travaux de recherche.

Ainsi, dans (Ferdousi et al., 2018 ; s. d.), nous avons proposé une approche de pré-filtrage à base de corrélations (CBPF), qui intègre des informations contextuelles dans le processus de recommandation en modélisant l'influence du contexte sur les scores. Cette influence est calculée par le coefficient de corrélation de Pearson (à partir des éléments recommandables ou des utilisateurs) entre un contexte et des scores. CPBF tente de tirer avantage des informations contextuelles dans son processus de recommandation pour améliorer ses performances.

Dans notre analyse expérimentale, nous avons évalué cette approche sur trois jeux de données contextuels différents très utilisés dans la communauté des systèmes de recommandation contextuels ((a) CoMoDa, pour la recommandation de films, dont les données ont été recueillies à partir d'enquêtes (Košir et al., 2011), (b) STS (Braunhofer et al., 2013), dans le domaine du tourisme, contenant des évaluations contextuelles de lieux d'intérêt, collectées à l'aide d'une application de tourisme mobile, et (c) Music, pour la recommandation de musique, dont les données ont été collectées par un système de recommandation musicale développé par (Baltrunas et al., 2011)). Nos expériences ont permis de valider l'effet positif de la prise en compte d'informations contextuelles sur l'utilisateur dans le processus de recommandation et de montrer que notre approche surpasse les techniques existantes dans la plupart des cas. De plus, les résultats expérimentaux montrent que le coefficient de corrélation de Pearson peut saisir efficacement l'influence du contexte sur les scores. Enfin, en raison du grand nombre d'éléments/utilisateurs, leur regroupement réduit non seulement les coûts de calcul, mais améliore également les performances.

A l'heure actuelle, nous n'avons pas pu tester cette approche (générique) de système de recommandation contextuel dans le cas de la vente de véhicules d'occasion, mais les résultats obtenus sur trois jeux de données réels différents semblent prometteurs. 


\subsection{En gestion de crises}

Dans le cadre de la gestion de crises, les informations contextuelles sont nombreuses. Celles relatives à l'individu peuvent être obtenues via son comportement, c'est-à-dire qu'elles sont collectées implicitement. Nous nous sommes donc intéressés au comportement des populations. Ainsi, nous avons mis en adéquation les concepts de comportement et de contexte.

\subsubsection{Comportement}

Le comportement est un concept qui nécessite d'être précisé et bien défini. Nos travaux s'appuient sur la définition proposée par (Sillamy, 1983) pour qui le comportement correspond aux « réactions d'un individu, considéré dans un milieu et dans une unité de temps donnée à une excitation ou un ensemble de stimulations ». Cette définition permet de situer clairement le comportement dans un espace spatiotemporel et comme une réponse à un ensemble d'excitations ou stimulations. Notons que nous limitons l'étude des comportements aux réactions observables par une entité extérieure.

Dans (Arru, Negre, 2017), nous avons décrit les comportements individuels en décomposant le plus finement possible les différents facteurs orientant les comportements, à l'aide d'éléments mesurables, les indicateurs.

Nous avons ainsi détecté vingt facteurs (et environ 70 indicateurs associés) du comportement, ayant une influence sur les réactions humaines en situation de crise.

\subsubsection{Familles et unités de contexte}

Nous présentons les facteurs de comportement selon la catégorisation des facteurs/unités de contexte présentée précédemment.

\subsubsection{Contexte Physique}

- Unité temporelle : les caractéristiques de la période pendant laquelle survient la crise (jour/nuit, ...) et la phase temporelle de la crise (avant, au commencement, ...)

- Unités spatiale et environnementale : les caractéristiques de la zone géographique (étendue de la zone, densité de la population, ...).

- Unité Équipement : la capacité d'interaction et de mobilité (fréquentation de la zone, nombre de smartphones par habitant, accès aux transports, ...).

\subsubsection{Contexte Personnel}

- Unité démographique : l'état civil (âge, sexe, lieu de résidence, ...)

- Unité psychophysiologique : la personnalité (désirs, principes moraux, sociabilité, ...), la motivation à évacuer/à défendre, les émotions (joie, tristesse, colère, ...) et les signaux physiologiques (rythme cardiaque, tension, niveau de transpiration, ...). 
- Unité sociale : le niveau de responsabilité, les caractéristiques de l'entourage (densité des personnes à proximité de l'individu, présence de représentants de l'autorité, ...), le comportement des personnes les plus proches, et le comportement global de l'entourage.

- Unité cognitive : l'expérience et les capacités, les connaissances explicitées, l'évaluation du risque, la perception du système d'alertes et les actions courantes.

\subsubsection{Contexte Technique}

- Unité Données : les signaux perceptibles de la crise (visuels, sonores et olfactifs) et les alertes/ informations transmises (quantité, qualité, ...).

Finalement, ici, chaque facteur de comportement est associé à une unité de contexte (une unité pouvant regrouper plusieurs facteurs de comportement).

\subsubsection{Modélisation}

Dans le cadre de la gestion de crise, modéliser le contexte en intégrant nos facteurs donne la possibilité de prendre en compte les réactions humaines. Avec un tel modèle, il est possible de tester des hypothèses sur la participation des différents facteurs aux comportements de crise pour une population donnée. Il est également possible de distinguer l'importance des différents facteurs impliqués pour différents types de crise. Les résultats obtenus à partir de ces analyses pourraient aider à la prévention et à la préparation des programmes de gestion des crises et aider à apporter des modèles qui fournissent des prédictions en temps réel. Une combinaison de différents modèles semble nécessaire à la fois pour représenter les définitions et la complexité de l'interaction, et pour pouvoir agréger les données pour offrir une vision synthétique des hypothèses validées. Beaucoup d'approches proposent déjà des combinaisons de plusieurs modèles (Lin, Lee, 1991; Ding et al., 2006; Swat et al., 2016). Nous avons retenu trois choix qui peuvent intégrer nos facteurs de comportement dans des objectifs d'analyse mathématique ou sémantique, les modèles attribut-valeur, les ontologies et les modèles prédictifs dans (Arru, Negre, 2017) :

- modèles attribut-valeur pour une analyse statistique : un modèle attribut-valeur peut être directement extrait de nos indicateurs. Selon l'analyse choisie au préalable, nous pouvons sélectionner les indicateurs et corrélations à tester. La méthode ACM (Analyse des Correspondances Multiples) pourrait être particulièrement adaptée pour tester des hypothèses à partir de ce type de modèle, elle permet de traiter les liens de corrélation entre n'importe quel nombre de variables. Elle est très souvent utilisée dans l'analyse de réponses à des questionnaires. En pratique, nous pouvons utiliser cette méthode pour expliquer un ou plusieurs indicateurs à partir d'un autre ensemble d'indicateurs. Malheureusement, cette méthode ne permet pas de reconnaitre les relations déjà connues entre indicateurs.

- ontologies et règles de décision : des ontologies ont déjà été proposées pour représenter des stratégies comportementales (Silverman, 2001; Silverman et al., 2006) ainsi que des informations contextuelles (Soualah Alila, 2015). Il est possible d'intégrer nos facteurs de comportement en implémentant une ontologie, et en y ajoutant un module de mémoire de travail qui permettrait de tirer avantage de règles de décisions pour découvrir de l'information à partir de données collectées.

- modèles prédictifs et analyses de corrélation : en partant d'une information complète sur des situations passées ou des études statistiques, nous pourrions construire un réseau Bayésien ou des modèles de processus markoviens qui permettraient de réaliser des prédictions à partir de données 
existantes dans les systèmes de gestion de crise et de tables de probabilités. Aujourd'hui, nous n'avons pas cette possibilité. Les réseaux Bayésiens sont à la fois (i) des modèles de représentation des connaissances (ii) des calculateurs de probabilités conditionnelles, et (iii) une base pour l'aide à la décision. Ici, nous pourrions décrire les relations causales entre variables à partir de ce type de graphe. Les relations causales entre les variables ne sont pas déterministes mais bien probabilistes. Par conséquent, l'observation d'une cause n'implique pas systématiquement les effet(s) qui dépendent d'elle, mais elle modifie la probabilité de les observer. L'intérêt d'utiliser des réseaux Bayésiens est de tenir compte simultanément des connaissances a priori (représentées par le graphe), et de l'expérience apportée par les données.

A première vue, une combinaison de ces trois modèles, combinant connaissance, expertise, expérience et technologie/ingénierie statistique, pourrait être envisagée, permettant de bénéficier de chacune, obtenant ainsi la modélisation la plus complète et aussi la plus réaliste possible.

Dans (Arru, Negre, 2017), les vingt facteurs de comportement ont été organisés selon deux classes : les facteurs liés à l'individu et ceux liés à l'environnement (comme illustré sur la figure 5a). Passer à notre typologie (comme illustré sur la figure 5b) permettrait de modéliser les facteurs à différents niveaux et ainsi réaliser des analyses plus poussées. Par exemple, des analyses des comportements par famille/unité de contexte (trois familles et dix unités) seraient peut-être plus pertinentes qu'une analyse essayant de corréler les vingt facteurs ensembles ou selon deux classes.

\subsubsection{Intégration}

L'urgence d'une situation de crise exige que les décisions qui conduisent à sa résolution soient rapides et efficaces (Altemaire, Renaudin, 2007). Dans une cellule de crise, les décisions sont conditionnées par de grandes incertitudes, un grand nombre de parties prenantes, des durées extrêmement courtes ou relativement longues, des problèmes de communication et des questions importantes bien au-delà des aspects opérationnels immédiats (Lagadec, 1995). Qu'elles fassent ou non partie des plans d'urgence, de nombreuses décisions doivent être prises. Ces décisions sont généralement prises de manière collective et portent sur le choix des actions à mener et des ressources allouées à ces actions. Les décisions sont prises par une multiplicité de parties prenantes, ce qui peut créer des difficultés pour trouver un terrain d'entente pour toutes les parties prenantes. Les décisions ne font pas toujours consensus. Ainsi, des outils pour aider à prendre des décisions sont nécessaires.

Au vu des enjeux de la prise de décision en gestion de crise, automatiser le processus de décision, via, par exemple, un système de recommandation, semble prématuré. En effet, les décideurs ont besoin de comprendre pourquoi et comment le système de recommandation en est arrivé à de telles propositions. Malheureusement, les travaux de recherche dans le domaine de l'explicabilité et de la transparence des algorithmes dans les systèmes de recommandation ou plus largement en Machine learning, ne sont pas suffisamment avancés pour répondre aux questions des décideurs en gestion de crise.

De plus, ces deux derniers nécessitent de gros volumes de données pour apprendre un modèle et vérifier son applicabilité. En gestion de crise, un tel volume de données relatives à de nombreuses crises "similaires" est actuellement inexistant. Il faut donc se tourner vers des modèles d'aide à la décision capables de travailler avec de petits ensembles de données et permettant, en outre, d'avoir quelques explications sur les décisions proposées. Pour rendre ces informations accessibles aux décideurs qui ne 


\begin{tabular}{|c|c|c|}
\hline \multirow{20}{*}{ Comportements } & \multirow{11}{*}{$\begin{array}{l}\text { Facteurs liés à } \\
\text { l'individu }\end{array}$} & Etat civil \\
\hline & & Personnalité \\
\hline & & Motivation à s'enfuir/se défendre \\
\hline & & Émotions \\
\hline & & Signaux physiologiques \\
\hline & & Responsabilité \\
\hline & & Expérience \\
\hline & & Connaissances explicitées \\
\hline & & Évaluation du risque \\
\hline & & Perception du système d'alerte \\
\hline & & Action courante \\
\hline & \multirow{9}{*}{$\begin{array}{c}\text { Facteurs liés à } \\
\text { l'environnement }\end{array}$} & Caractéristiques de la période \\
\hline & & Phase temporelle de la crise \\
\hline & & Caractéristiques de la zone géographique \\
\hline & & Capacité d'interaction \\
\hline & & Caractéristiques de l'entourage \\
\hline & & Comportement des personnes les plus proches \\
\hline & & Comportement global de l'entourage \\
\hline & & Signaux perceptibles de la crise \\
\hline & & Alertes / informations transmises \\
\hline
\end{tabular}

(a) Classification de (Arru, Negre, 2017)

\begin{tabular}{|c|c|c|c|}
\hline & Famille & Unité & Facteur \\
\hline \multirow{21}{*}{$\begin{array}{c}\text { Contexte - } \\
\text { Comportement }\end{array}$} & \multirow{5}{*}{ Contexte physique } & \multirow{2}{*}{ Temporelle } & Caractéristiques de la période \\
\hline & & & Phase temporelle de la crise \\
\hline & & Spatiale & Caractéristiques de la zone géographique \\
\hline & & Environnementale & Caractéristiques de la zone géographique \\
\hline & & Équipement & Capacité d'interaction \\
\hline & \multirow{14}{*}{ Contexte personnel } & Démographique & Etat civil \\
\hline & & \multirow{4}{*}{ Psychophysiologique } & Personnalité \\
\hline & & & Motivation à s'enfuir/se défendre \\
\hline & & & Émotions \\
\hline & & & Signaux physiologiques \\
\hline & & \multirow{4}{*}{ Sociale } & Responsabilité \\
\hline & & & Caractéristiques de l'entourage \\
\hline & & & Comportement des personnes les plus proches \\
\hline & & & Comportement global de l'entourage \\
\hline & & \multirow{5}{*}{ Cognitive } & Expérience \\
\hline & & & Connaissances explicitées \\
\hline & & & Évaluation du risque \\
\hline & & & Perception du système d'alerte \\
\hline & & & Action courante \\
\hline & \multirow{2}{*}{ Contexte technique } & \multirow{2}{*}{ Données } & Signaux perceptibles de la crise \\
\hline & & & Alertes / informations transmises \\
\hline
\end{tabular}

(b) Les facteurs de comportement vus comme des facteurs de contexte selon notre typologie

Figure 5. Facteurs de comportement. 
sont pas des informaticiens mais qui prennent des décisions avec des problèmes majeurs, une possibilité consiste à utiliser des arbres de décision (Quinlan, 1987) qui ont la spécificité de représenter un ensemble de choix, sous la forme graphique d'un arbre. Les différentes décisions possibles se situent aux extrémités des branches (les "feuilles" de l'arbre) et sont atteintes en fonction des décisions prises à chaque étape. L'arbre de décision a l'avantage d'être facile à lire et rapide à exécuter (Quinlan, 1987).

Ainsi, dans (Arru et al., 2019), nous fournissons une méthode d'analyse de données qui permet aux décideurs des cellules de crise d'avoir des éléments de réponse à la question d'alerter ou non les populations dans une zone géographique donnée. Cette méthode est basée sur une sélection de facteurs/indicateurs qui influencent les comportements de la population, comme présentés précédemment via notre typologie de contexte. Enfin, nous proposons un outil d'aide à la décision (basé sur un arbre de décision en tant que représentation possible). La figure 6 montre un arbre de décision simple (obtenu avec l'algorithme Random Tree et quatre décisions possibles : panique, rumeurs, panique-et-rumeurs, rien (pas de réaction de panique ni de rumeur).) pouvant être proposé à un décideur dans une cellule de crise. Par exemple, ici, si une alerte est lancée entre $14 \mathrm{~h}$ et $18 \mathrm{~h}$ pour une population dont la proportion de plus de 75 ans est supérieure ou égale à $7.5 \%$ alors, il y a un fort risque de panique de la population face à cette alerte.

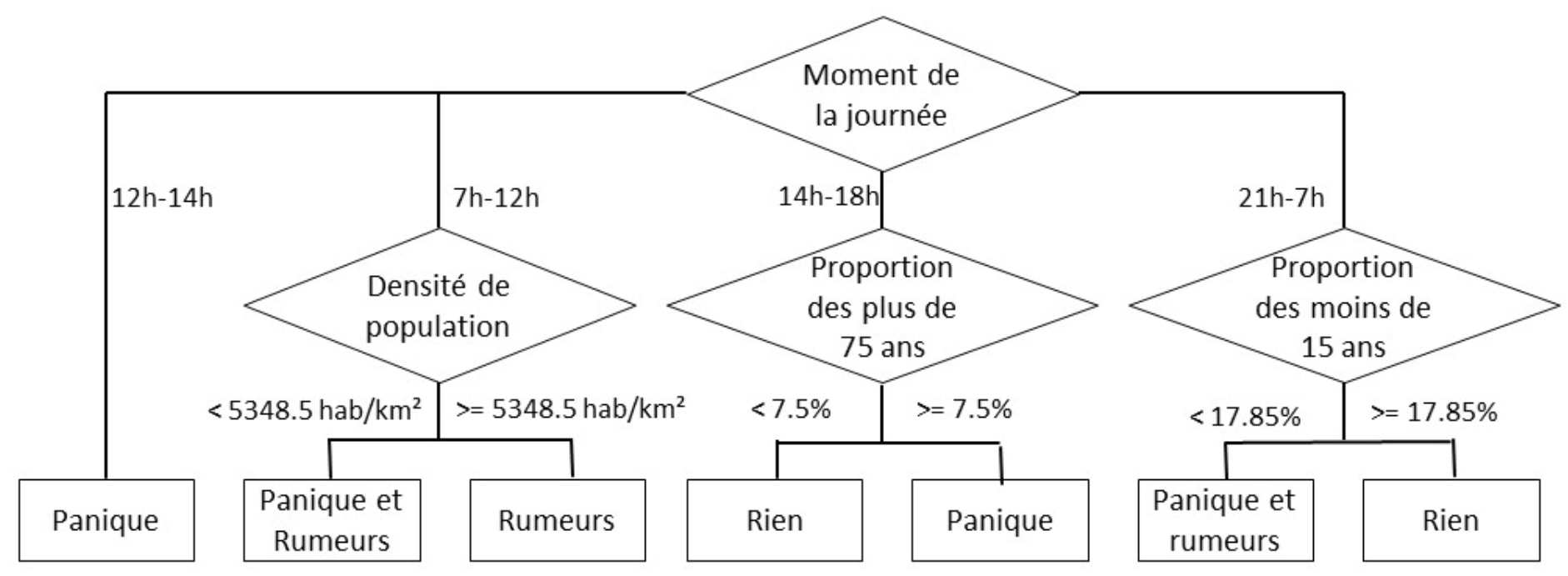

Figure 6. Arbre de décision obtenu avec l'algorithme Random Tree et quatre décisions possibles : panique, rumeurs, panique-et-rumeurs, rien (pas de réaction de panique ni de rumeur). L'absence de branche relative à 18h-21h est due à l'absence de donnée sur ce moment de la journée.

\section{Conclusion}

Dans cet article, nous proposons une typologie de contexte pour les systèmes de recommandation afin d'être au plus près des besoins de l'utilisateur et améliorer les recommandations. Cette typologie de contexte est présentée selon trois familles et dix unités de contexte. Elle a été instanciée en OLAP, dans un environnement commercial (de ventes de véhicules d'occasion) et en gestion de crise (via les comportements humains).

Le tableau 2 récapitule les informations contextuelles détectées comme pertinentes pour chaque cas d'application.

Nos propositions montrent ainsi quelles informations contextuelles peuvent être prises en compte dans le processus de décision, comment les modéliser et comment les intégrer dans un système de recom- 


\begin{tabular}{|c|c|c|c|c|}
\hline Contexte & Unité & OLAP & $\begin{array}{c}\text { Commerce } \\
\text { (véhicules d'occasion) }\end{array}$ & $\begin{array}{c}\text { Gestion de crise } \\
\text { (comportements) }\end{array}$ \\
\hline \multirow{3}{*}{ Physique } & Temporelle & $\checkmark$ & $\checkmark$ & $\checkmark$ \\
& Spatiale & & $\checkmark$ & $\checkmark$ \\
& Environnementale & & $\checkmark$ & $\checkmark$ \\
& Équipement & & $\checkmark$ & $\checkmark$ \\
\hline \multirow{3}{*}{ Personnel } & Démographique & $\checkmark$ & $\checkmark$ & $\checkmark$ \\
& Psychophysiologique & & $\checkmark$ & $\checkmark$ \\
& Sociale & $\checkmark$ & $\checkmark$ & $\checkmark$ \\
\hline Technique & Cognitive & $\checkmark$ & & $\checkmark$ \\
& Matériel & $\checkmark$ & & \\
\hline
\end{tabular}

Tableau 2. Récapitulatif des informations contextuelles pertinentes selon les domaines/cas d'applications

mandation (qui devient contextuel). Ces données/sources externes permettront de mieux connaître l'utilisateur, d'enrichir son profil, d'améliorer la pertinence des recommandations, de densifier les données d'entrée avec des données complémentaires et ainsi d'améliorer les recommandations.

Pouvoir utiliser notre typologie ${ }^{8}$ dans trois domaines différents, sans compromettre ni la modélisation du contexte, ni son intégration au sein d'un système de recommandation (contextuel), valide sa généricité et son applicabilité. Il est à noter que nos tentatives d'implémentation et expérimentations réalisées sont prometteuses.

Par ailleurs, de nouvelles idées commencent à émerger dans le domaine des systèmes de recommandation (contextuels). En voici quelques exemples :

- (Pagano et al., 2016) ont récemment initié l'idée selon laquelle la prochaine évolution des systèmes de recommandation serait de passer d'un système de recommandation contextuel (context-aware) qui s'adapte au contexte à un système de recommandation piloté par le contexte (context-driven). L'idée est de ne plus prendre en compte le contexte comme une information complémentaire, mais de le faire passer à l'avant plan, c'est-à-dire faire de la recommandation en se basant sur ce qui se passe autour de l'utilisateur à l'instant donné (la situation), et ce qu'il est en train d'accomplir (l'intention), au lieu de se baser sur son comportement dans le passé. Cet axe de recherche soulève de nombreux défis liés notamment à la modélisation et à l'intégration d'information (contextuelle) continue/temps réel sans endommager sa valeur, et à la sérialité des éléments (il existe une dépendance séquentielle entre les éléments puisque le contexte est une information continue).

- La prolifération des sites de commerce électronique, des médias sociaux, ... a permis aux utilisateurs de fournir des commentaires, d'exprimer leurs préférences/intérêts et de maintenir des profils utilisateurs dans de multiples systèmes, reflétant la variété de leurs goûts/intérêts. Tirer parti de toutes ces informations disponibles dans différents systèmes et relatives à différents champs/spécialités peut être bénéfique pour générer des profils utilisateurs plus complets et de meilleures recommandations, par exemple, en proposant des recommandations personnalisées « croisées » pour des éléments de champs différents. Les systèmes de recommandation multi-domaines (cross-domain recommender systems) visent à générer ou à améliorer des recommandations pour un champs particulier en exploitant les profils utilisateurs (ou toutes autres données/informations) issues d'autres

8. Il est à noter que notre typologie est actuellement "hors sol". Nos travaux futurs viseront à étudier la possibilité de la rattacher à des ontologies comme celles de (Wang et al., 2004) ou (Schmidt, 2006) 
champs (Ricci et al., 2011). Cet axe de recherche émergent soulève de nombreux défis comme la définition de la tâche/stratégie de tels systèmes ou encore les techniques/modèles appropriés pour le transfert des données/informations/connaissances d'un champs à un autre, ... (Khan et al., 2017). Notre proposition de typologie de contexte est un pas en ce sens.

\section{Bibliographie}

Adomavicius G., Tuzhilin A. (2011). Context-aware recommender systems. In F. Ricci, L. Rokach, B. Shapira, P. B. Kantor (Eds.), Recommender systems handbook, p. 217-253. Boston, MA, Springer US.

Akermi I., Boughanem M., Faiz R. (2015). Une approche de recommandation proactive dans un environnement mobile. In Inforsid, p. 301-316.

Altemaire A., Renaudin H. (2007). Prendre les meilleures décisions dans un contexte de gestion de crise. Le magazine de la communication de crise et sensible, vol. 13.

Arduin P., Mayag B., Negre E., Rosenthal-Sabroux C. (2014). How to compromise on the best price? A group tacit knowledge-based multicriteria approach. Journal of Decision Systems, vol. 23, no 1, p. 99-112.

Arru M., Negre E. (2017). People behaviors in crisis situations : Three modeling propositions. In Information systems for crisis response and management - iscram, albi, may 2017.

Arru M., Negre E., Rosenthal-Sabroux C. (2019). To alert or not to alert? that is the question. In 52th hawaii international conference on system sciences, HICSS 2019, hawaii, usa, january 8-11, 2019.

Baltrunas L., Kaminskas M., Ludwig B., Moling O., Ricci F., Aydin A. et al. (2011). Incarmusic : Context-aware music recommendations in a car. In C. Huemer, T. Setzer (Eds.), E-commerce and web technologies, p. 89-100. Berlin, Heidelberg, Springer Berlin Heidelberg.

Baltrunas L., Ludwig B., Peer S., Ricci F. (2012, juin). Context relevance assessment and exploitation in mobile recommender systems. Personal Ubiquitous Comput., vol. 16, no 5, p. 507-526.

Bazire M., Brézillon P. (2005). Understanding context before using it. In Proceedings of the 5th international conference on modeling and using context, p. 29-40. Berlin, Heidelberg, Springer-Verlag.

Benouaret I. (2015). Un système de recommandation sensible au contexte pour la visite de musée. In CORIA 2015 - conférence en recherche d'infomations et applications - 12th french information retrieval conference, paris, france, march 18-20, 2015., p. 515-524.

Bettini C., Brdiczka O., Henricksen K., Indulska J., Nicklas D., Ranganathan A. et al. (2010). A survey of context modelling and reasoning techniques. Pervasive and Mobile Computing, vol. 6, n ${ }^{\circ}$ 2, p. 161 - 180. (Context Modelling, Reasoning and Management)

Braunhofer M., Elahi M., Ricci F., Schievenin T. (2013). Context-aware points of interest suggestion with dynamic weather data management. In Z. Xiang, I. Tussyadiah (Eds.), Information and communication technologies in tourism 2014, p. 87-100. Cham, Springer International Publishing.

Brown P. J., Bovey J. D., Chen X. (1997). Context-aware applications : from the laboratory to the marketplace. IEEE Personal Commun., vol. 4, no 5, p. 58-64.

Burke R. D., Hammond K. J., Young B. C. (1997). The findme approach to assisted browsing. IEEE Expert, vol. 12, p. 32-40.

Cool C. (2001). The concept of situation in information science. Annual Review of Information Science and Technology, $\mathrm{n}^{\mathrm{o}} 35$, p. $5-42$.

Dey A., Salber D., Abowd G. (2001). A conceptual framework and a toolkit for supporting the rapid prototyping of context-aware applications.

Ding Z., Peng Y., Pan R. (2006). A bayesian approach to uncertainty modelling in owl ontology. Rapport technique. DTIC Document.

Ferdousi Z. V., Colazzo D., Negre E. (s. d.). Cbpf : leveraging context and content information for better recommendations. In The 14th international conference on advanced data mining and applications (adma), 16-18 november, 2018, nanjing, china. 
Ferdousi Z. V., Colazzo D., Negre E. (2018). Correlation-based pre-filtering for context-aware recommendation. In 2018 IEEE international conference on pervasive computing and communications workshops, percom workshops 2018, athens, greece, march 19-23, 2018, p. 89-94.

Ferdousi Z. V., Negre E., Colazzo D. (2017). Context factors in context-aware recommender systems. In Atelier interdisciplinaire sur les systèmes de recommandation.

Henricksen K., Indulska J. (2006, février). Developing context-aware pervasive computing applications : Models and approach. Pervasive Mob. Comput., vol. 2, nº 1, p. 37-64.

Ingwersen P., Järvelin K. (2005). The turn: Integration of information seeking and retrieval in context (the information retrieval series). Secaucus, NJ, USA, Springer-Verlag New York, Inc.

Khan M. M., Ibrahim R., Ghani I. (2017, juin). Cross domain recommender systems : A systematic literature review. ACM Comput. Surv., vol. 50, no 3, p. $36: 1-36: 34$.

Košir A., Odic A., Kunaver M., Tkalcic M., Tasic J. F. (2011). Database for contextual personalization. Elektrotehniški vestnik, vol. 78.

Lagadec P. (1995). Cellules de crise : Les conditions d'une conduite efficace, gouvernements, ministères, entreprises, préfectures, administrations, municipalités, régions, médias, organisations internationales, organisations non gouvernernentales, associations, syndicats. les Éd. d'Organisation.

Lin C.-T., Lee C. S. G. (1991). Neural-network-based fuzzy logic control and decision system. IEEE Transactions on computers, vol. 40, nº 12, p. 1320-1336.

Mostefaoui G. K., Pasquier-Rocha J., Brézillon P. (2004). Context-aware computing : A guide for the pervasive computing community. In Icps, p. 39-48. IEEE Computer Society.

Negre E. (2017). Prise en compte du contexte dans les systèmes de recommandation de requêtes olap. In Actes des journées francophones sur les entrepôts de données et l'analyse en ligne, EDA 2017, lyon, france.

Negre E. (2018). Vers une typologie de contexte pour les systèmes de recommandation. In Actes du xxxvième congrès inforsid, nantes, france, may 28-31, 2018, p. 197-212. Consulté sur http://inforsid.fr/actes/2018/INFORSID2018Negre .pdf

Negre E., Ravat F., Teste O. (2018). OLAP queries context-aware recommender system. In Database and expert systems applications - 29th international conference, DEXA 2018, regensburg, germany, september 3-6, 2018, proceedings, part II, p. 127-137.

Nguyen C. (2010). Conception d'un système d'apprentissage et de travail pervasif et adaptatif fondé sur un modèle de scénario. Thèse de doctorat non publiée, Ecole Nationale Supérieure des Télécommunications de Bretagne.

Pagano R., Cremonesi P., Larson M., Hidasi B., Tikk D., Karatzoglou A. et al. (2016). The contextual turn : From contextaware to context-driven recommender systems. In Proceedings of the 10th acm conference on recommender systems, $\mathrm{p}$. 249-252.

Panniello U., Gorgoglione M. (2011). Context-aware recommender systems : A comparison of three approaches. In Proceedings of the 5th international workshop on new challenges in distributed information filtering and retrieval, palermo, italy, september 17, 2011.

Panniello U., Tuzhilin A., Gorgoglione M., Palmisano C., Pedone A. (2009). Experimental comparison of pre- vs. postfiltering approaches in context-aware recommender systems. In Proceedings of the third acm conference on recommender systems, p. 265-268. New York, NY, USA, ACM.

Petrelli D., Not E., Strapparava C., Stock O., Zancanaro M. (2000). Modeling context i s like taking pictures. In Conference on human factors in computers, workshop "the what, who, where, when, why and how of context-awareness".

Quinlan J. R. (1987). Generating production rules from decision trees. In Proceedings of the 10th international joint conference on artificial intelligence - volume 1, p. 304-307. San Francisco, CA, USA, Morgan Kaufmann Publishers Inc.

Riboni D., Bettini C. (2011, mars). Cosar : Hybrid reasoning for context-aware activity recognition. Personal Ubiquitous Comput., vol. 15, n 3, p. 271-289.

Ricci F., Rokach L., Shapira B., Kantor P. B. (Eds.). (2011). Recommender systems handbook. Springer.

Schilit B., Theimer M. (1994, sep/oct). Disseminating active map information to mobile hosts. Network, IEEE, vol. 8, nº 5, p. $22-32$. 
Schmidt A. (2006). Ontology-based user context management : The challenges of imperfection and time-dependence. In R. Meersman, Z. Tari (Eds.), On the move to meaningful internet systems 2006 : Coopis, doa, gada, and odbase, p. 995-1011. Springer Berlin Heidelberg.

Sillamy N. (1983). Dictionnaire usuel de psychologie. Bordas.

Silverman B. G. (2001). More realistic human behavior models for agents in virtual worlds : emotion, stress, and value ontologies. ACASA Technical Report.

Silverman B. G., Johns M., Cornwell J., O’Brien K. (2006). Human behavior models for agents in simulators and games : part i : enabling science with pmfserv. Presence : Teleoperators and Virtual Environments, vol. 15, nº 2, p. 139-162.

Soualah Alila F. (2015). Camlearn* : une architecture de système de recommandation sémantique sensible au contexte : application au domaine du m-learning. Thèse de doctorat non publiée. (Dijon)

Swat M. J., Grenon P., Wimalaratne S. (2016). Probonto-ontology and knowledge base of probability distributions. Bioinformatics.

Wang X. H., Zhang D. Q., Gu T., Pung H. K. (2004). Ontology based context modeling and reasoning using owl. In Proceedings of the second ieee annual conference on pervasive computing and communications workshops, p. 18-. IEEE Computer Society. 